Methods We enrolled 1,200 patients and randomly allocated them into 4 groups with different types of premedication. Premedication used in regular group (RG) was $10 \mathrm{~mL}$ lidocaine hydrochloride mucilage (LHM) and in pronase+simethicone group $(\mathrm{P}+\mathrm{S})$ was $60 \mathrm{~mL}$ pronase/simethicone solution plus 10 mL LHM. Meanwhile, $60 \mathrm{~mL}$ NAC600 mg/simethicone solution plus $10 \mathrm{~mL}$ LHM and $60 \mathrm{~mL}$ NAC1200 mg/simethicone solution plus $10 \mathrm{~mL}$ LHM were given to the patients in NAC600+simethicone group (NAC600+S) and NAC1200 +simethicone group (NAC1200+S), respectively. We recorded visibility scores, times of cleaning, time taken for examination, diminutive lesions and pathological diagnosis. Cost-benefit of premedication was analyzed.

Results The visibility of NAC600+S and NAC1200+S are significantly better than $R G$, although $P+S$ exhibited the lowest total visibility score and shortest procedure duration (figure 1). Times of cleaning was remarkably decreased in NAC600 $+S$ and NAC1200+S compared to RG, but was inferior to $P$ $+S$. There is no significant difference in detection rate of diminutive lesions and pathological diagnosis. Cost-benefit analysis has shown that net medical yield of NAC/simethicone is higher than that of pronase/simethicone.

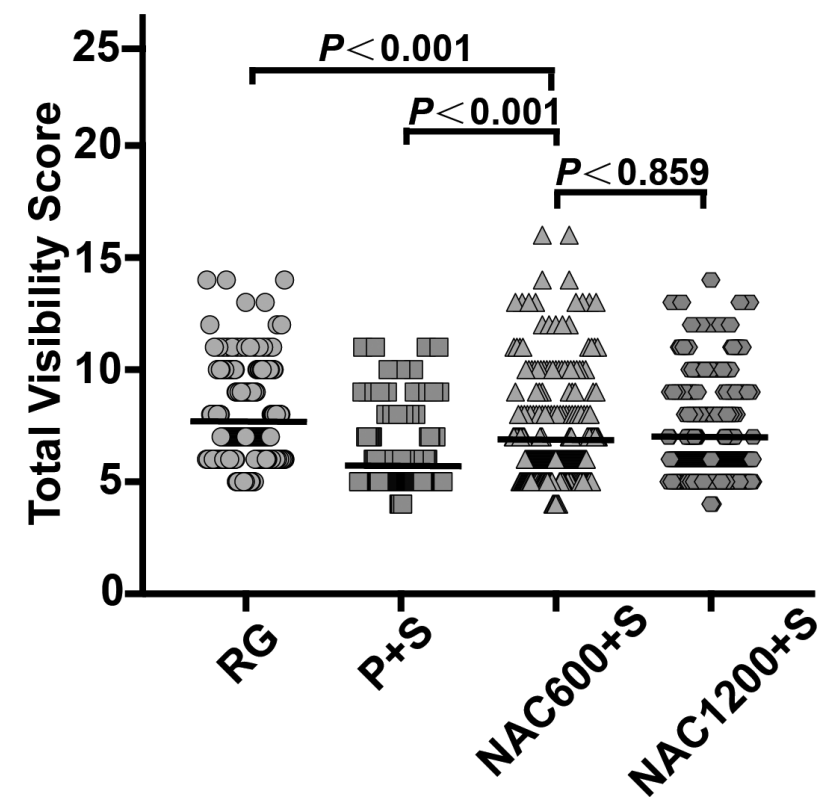

Abstract IDDF2019-ABS-0311 Figure 1 Total visibility score of each group

Conclusions NAC can not only facilitate the visibility during EGD, but be more cost-beneficial. It should be recommended as a good alternative premedication to pronase during EGD, especially in developing countries and regions.

\section{IDDF2019-ABS-0313 CLINICAL FACTORS AFFECTING RESPONSE TO FECAL MICROBIOTA TRANSPLANTATION IN PATIENTS WITH ACTIVE ULCERATIVE COLITIS}

Vandana Midha*, Ramit Mahajan, Arshdeep Singh, Varun Mehta, Yogesh Gupta. Dayanand Medical College and Hospital, Ludhiana, India

10.1136/gutjnl-2019-IDDFabstracts.230
Background Fecal microbiota transplantation (FMT) has emerged as a novel therapy for ulcerative colitis (UC). However patient selection in published trials is not uniform. Also, not all patients receiving FMT show a clinical response. Hence ideal patient population to be considered for FMT is not known. We study the factors affecting response to fecal microbiota transplantation in patients with active ulcerative colitis.

Methods This was a single-centre analysis of data from patients with active UC treated with FMT from September 2015 to December 2018 at the Dayanand Medical College, a tertiary care centre in India. Fecal samples from two unrelated donors [A and B, alternatively for each session (A.B.A.B.A.B. A)], were administered through colonoscopy at weeks $0,2,6$, 10, 14, 18, and 22. Disease activity including endoscopic severity (using Mayo score)and response to therapy was assessed and recorded at each visit. Clinical response was defined as a decrease from baseline in mayo clinic score by $\geq$ $30 \%$ and $\geq 3$ points with a decrease in rectal bleeding subscore of $\geq 1$ or rectal bleeding subscore of 0 or 1 . Both disease characteristics and patient characteristics affecting response to FMT were then retrospectively analysed.

Results One hundred twenty-four patients [mean age 34.84 \pm 11.91 years, $66.93 \%$ males $(n=83)$, mean mayo clinic score $8.13 \pm 2.65$, mean disease duration $5.04 \pm 4.82$ years] who received FMT were analysed retrospectively. The characteristics favouring response to FMT were E2 disease $(\mathrm{p}=0.01)$ and severe disease (greater total mayo clinic score, endoscopic mayo clinic score, ESR and CRP; $p=0.001,0.003,0.002$, 0.001 respectively). Low serum albumin was a predictor of non-response $(p=0.003)$. Patients with no pseudopolyps $(p=0.06)$ and previous exposure to biologics $(p=0.08)$ and thiopurines $(p=0.06)$ showed a trend towards response with FMT, however, failed to reach statistical significance. Concomitant use of corticosteroids did not affect the response rates with FMT $(\mathrm{p}>0.05)$.

Conclusions Left-sided UC, severe disease and high serum inflammatory markers (ESR and CRP) significantly predict response to FMT. Absence of pseudopolyps and previous exposure to biologics and thiopurines also favoured response.

\section{IDDF2019-ABS-0319 THE DIAGNOSTIC ACCURACY OF PAPANICOLAU STAINING IN BRUSH BIOPSY AS AN ALTERNATIVE TO TISSUE BIOPSY IN PATIENTS UNDERGOING COLONOSCOPY FOR NON-OBSTRUCTING COLONIC TUMORS}

Clint Becaldo* , Arlene Kuan. Vicente Sotto Memorial Medical Center, Cebu City, Philippines

\subsection{6/gutjnl-2019-IDDFabstracts.231}

Background The study was conducted to determine the diagnostic accuracy of papanicolau stain in detecting malignancy from brush biopsy specimens in patients undergoing colonoscopy.

Methods A prospective, open-label 19-month study, covering February 2016- September 2017 of patients aged 19 years and above. Demographics and informed consent of all patients undergoing colonoscopy were taken. On colonoscopy, brush biopsies around the four sides of tumors were sampled after the tissue biopsy then smeared to slides, fixed with $95 \%$ ethyl alcohol and stained with Papanicolau method and read by $\underline{2}$ independent pathologists. 